\title{
A COMPARISON OF EXCIMER LASER, THERMAL PROBE, AND MECHANICAL DEVICES FOR RECANALIZING OCCLUDED HUMAN ARTERIES
}

\author{
Masahito Moriuchi, M.D., Jonathan M. Tobis, M.D., Michael Mcrae, M.D. \\ John A. Mallery, M.D., Lachlan Macleay, M.D., Omar Moussabeck, M.D. \\ Michael Berns, PH.D., and Walter L. Henry, M.D.
}

\begin{abstract}
To evaluate the mechanism of excimer laser recanalization and compare the results with those of laser-assisted thermal probe recanalization and mechanical recanalization, a total of 42 human atherosclerotic totally occluded arterial segments $(2-15 \mathrm{~cm}$ long) were recanalized by excimer laser with a 400-800 micron quartz fiber pulsed at $20 \mathrm{~Hz}$ with $50 \mathrm{~mJ} / \mathrm{mm}^{2}$ of energy $(\mathrm{n}=21)$, an Argon heated thermal probe at $10-12$ watts $(n=11)$, a guidewire directed through a $6 \mathrm{Fr}$ multipurpose catheter, or an angioplasty balloon catheter $(n=10)$.

On histologic examination, the excimer laster created a single round lumen or multiple lumens ("Swiss-cheese" like appearance) with no evidence of thermal injury at the perimeter of the lumen. The incidence of perforation in vitro was less with an excimer laser catherter $(8 / 21$ or $38 \%)$ than with the thermal prove $(10 / 11$ or $91 \%)(p<0.01)$. However, serial histologic cross-sectional examination showed that the pathway of the devices were essentially the same in all recanalization procedures. The pathway of the device was located outside the atheroma but proximal to the internal elastic membrane in 13 arteries with the excimer laser $(62 \%)$, in 10 arteries with the thermal probe $(91 \%)$, and 8 arteries with mechnical devices $(80 \%)$.

These results indicate that although the eximer laser could recanalize human atherosclerotic arteries without thermal injury, the fiber frequently deflected around firm atherosclerotic plaque and advanced in a dissection plane between the plaque and media. A similar course was noted for the thermal probe or during mechanical recanalization with a guidewire and catheter. To insure the safety of an excimer fiber or a thermal probe to reopen complete occlusions, better guidance systems must be developed.
\end{abstract}

$\mathbf{L}$ ASER energy can ablate atherosclerotic tissue in vitro and recent clinical trials have shown the use of laser energy to be an effective means of recanalizing atherosclerotic occlusions. The clinical trials have in-

Key words:

Excimer laser angioplasty

Thermal probe angioplasty

Mechanical recanalization

Supported in part by NIH Grant \#44512229843 cluded a thermal (hot tip) metal probe, a bare Argon laser fiber, and an excimer laser catheter!-5 Each method has its own potential advantages and disadvantages, however, the most significant complication for any of the methods has been perforation of the artery. The precise mechanism of ablation of atherosclerotic tissue is not completely understood, although it is thought to involve thermal injury and vaporization of

(Received July 2, 1990; accepted October 3, 1990)

*The 2nd Department of Internal Medicine, Nihon University, School of Medicine, Tokyo, Japan

University of California, Irvine, Division of Cardiology and Pathology, Long Beach Veterans Adiministration Hospital, Department of Pathology, and the Beckman Laser Institute, Irvine, California

Mailing address: Jonathan M. Tobis, M.D., Division of Cadiology, UCI Medical Center, P.O. Box 14091, Orange, CA92613-4091 


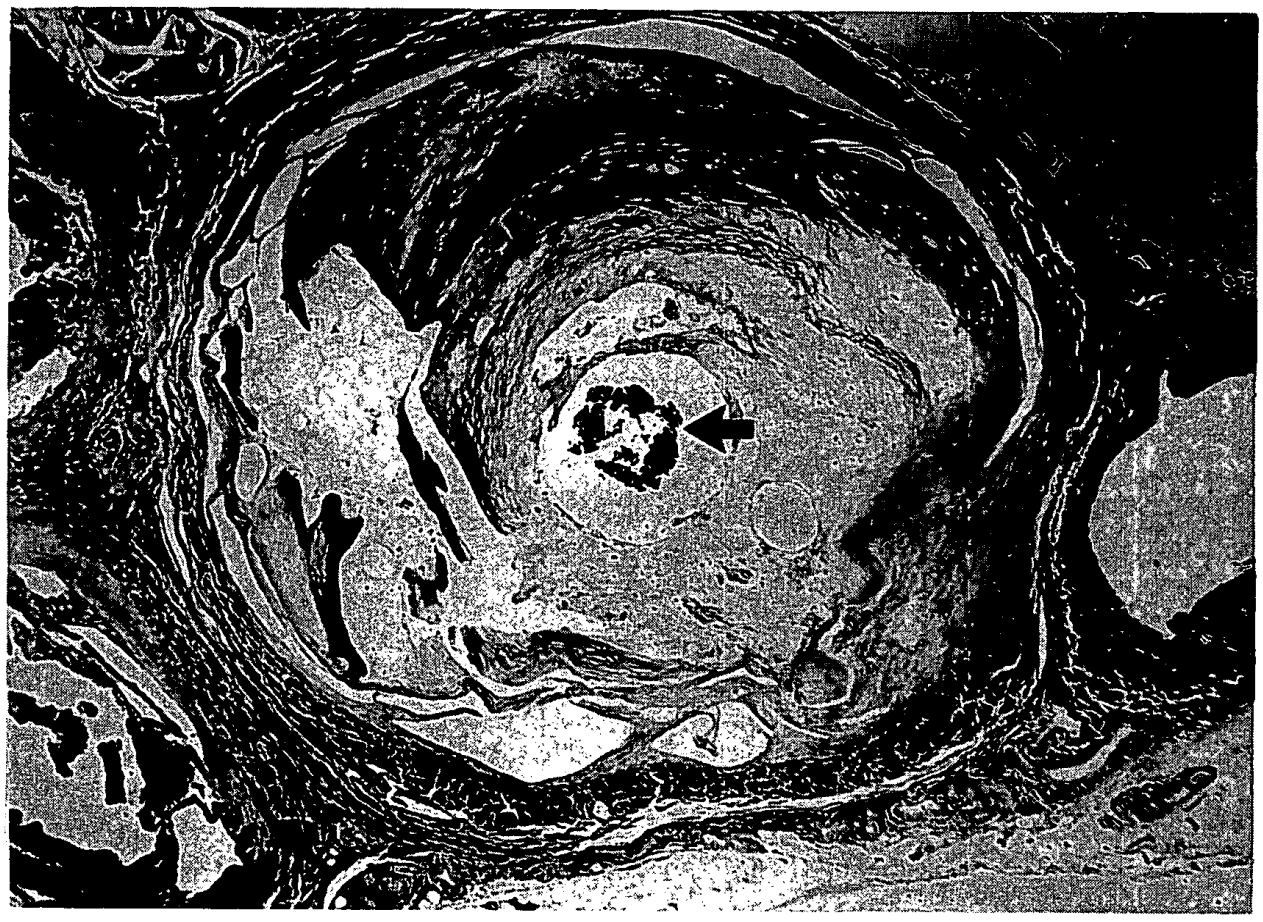

Fig.1. Cross sectional view of a totally occluded peripheral artery recanalized with the excimer laser. A newly created lumen, documented by the silk thread (arrow), is seen in the central portion of the atheroma. No significant thermal injury is noted at the perimeter of the lumen.

tissue through a complex interaction of laser energy with tissue proteins ${ }^{6-9}$ The exact method of recanalization of human atherosclerotic occlusions has not been clearly delineated, although it has generally been thought that the atherosclerotic plaque is ablated by laser radiation or the thermal effect with the result that a channel is created through the center of the occlusion. Recent investigators, however, suggest that the Argon Heated thermal peobe recanalizes occluded arteries predominantly through a mechanical process which is similar to the pathway created by standard guidewire and catheter techniques! ${ }^{10}$ The mechanism of action of the excimer laser is thought to be due to the interaction of the ultraviolet radiation with organic double bonds which produces destruction of tissue without creating a significant thermal injury to adjacent tissue ${ }^{11-13}$ Recent technical advancements in fiberoptic catheters have made it possible to use excimer laser radiation in human peripheral arteries and preliminary studies suggest that this method is effective and safe. -5

The purpose of the present study was to evaluate the mechanism of action during recanalization of totally occluded human arteries with an excimer laser catheter. In addition, the microscopic pathway of the excimer laser catheter was compared to the findings during mechanical recanalization with a multipurpose catheter and a guidewire, as well as to the pathway found during recanalization with an Argon Heated thermal probe.

\section{METHODS}

Tissue Sample A total of 42 totally occluded human (37 Caucasian and 5 Negro) atherosclerotic peripheral $(n=39)$ and coronary arteries $(n=3)$ were obtained at autopsy or from patients who had surgical amputation of the leg for symptoms of claudication with gangrene. The arteries were dissected from the leg or heart and stored in cold saline solution and used within one month. After transport to the laboratory, the arteries were cut into segments $2-15 \mathrm{~cm}$ in length. The mean occlusion length in the artery segments treated by the excimer fiber, thermal probe and mechanical guidewire were $3.6,6.1$ and $5.1 \mathrm{~cm}$, respectively. There was statistical significance between the length of occlusion treated with 


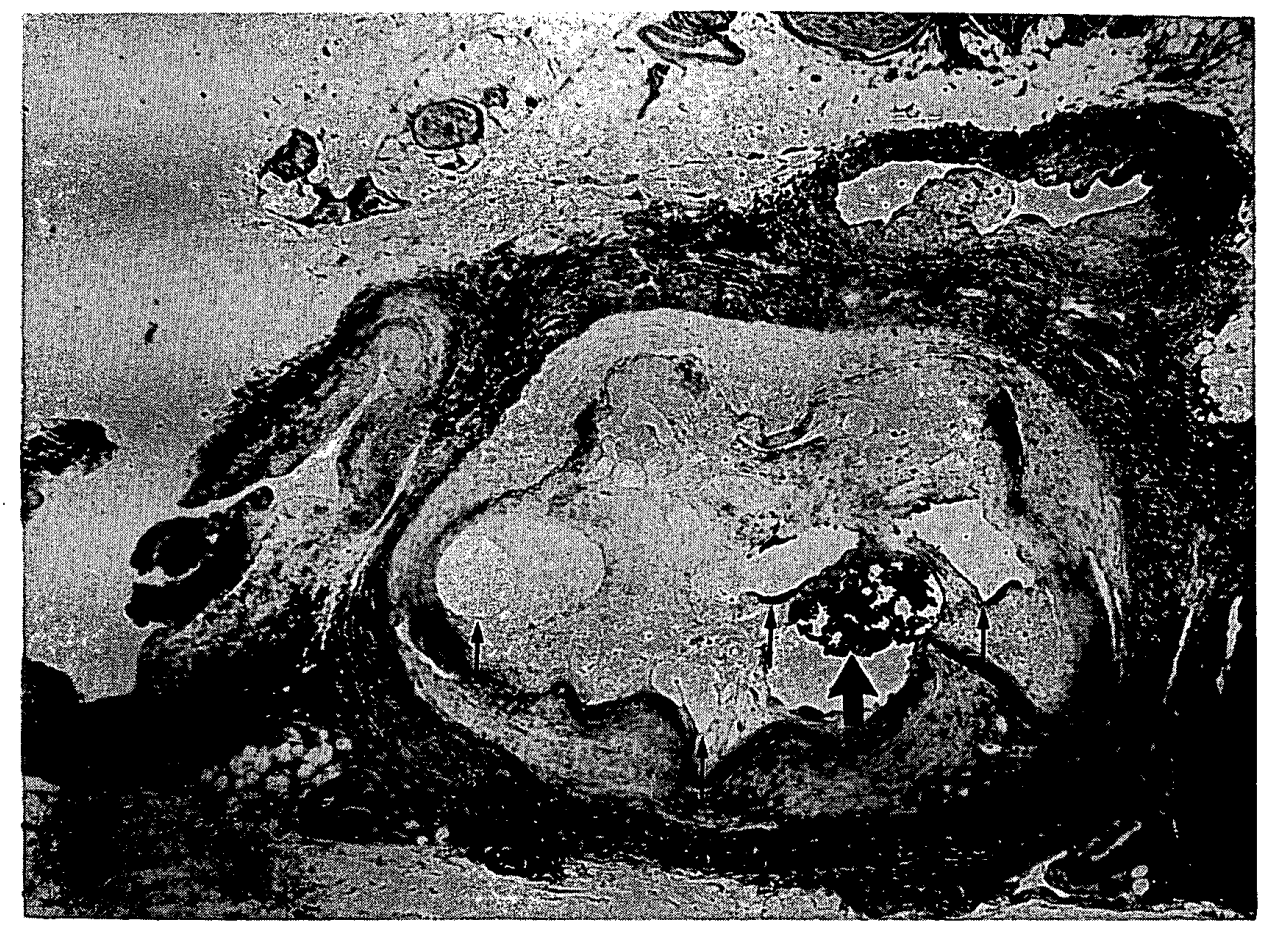

Fig.2. Cross-section of a totally occluded peripheral artery following excimer laser radiation showing multiple lumens (Swiss-cheese pattern, small arrows). All lumens are within the atheroma and no mechnical tears observed. One of the lumens is indicated by the silk thread (large arrow).

the excimer fiber and the other two methods (unpaired T-test, $\mathrm{p}<0.05$ ). The duration of occlusion could not be correctly identified because the arteries were found to be occluded at the time of autopsy; however, from the clinical status of the patients, it was concluded that all of the arteries had been occluded chronically, at least more than three months.

\section{RECANALIZATION PROCEDURES}

Excimer laser Excimer laser recanalization was performed on 21 artery segments. A xenon chloride excimer laser (Spectranetics, Colorado Springs, Colorado) at a wavelength of $308 \mathrm{~nm}$ and a $400-800$ micron quartz fiber were used in this study. The repetition rate and delivered energy were held constant as $20 \mathrm{~Hz}$ and $50 \mathrm{~mJ} / \mathrm{mm}^{2}$ with a pulse duration of 120 nanoseconds. The artery segment was pinned on a cardboard backing and the laser fiber was placed coaxially at the proximal site of the artery. The fiber was then advanced slowly with gentle forward pressure as the laser energy was delivered. The quarz fiber was glued to a 7 Fr. straight catheter which provided support. To isolate the effect of the excimer laser ablation from the mechanical shearing force of the supporting catheter, two arteries were recanalized with a bare quartz without the supporting catheter.

Thermal probe Laser-mediated thermal recanalization with the Laserprobe (Trimedyne Inc., Santa Ana, CA) was attempted in nine arterial segments and two arteries in situ during recanalization. The metal probe $(1.5-2.0 \mathrm{~mm})$ was heated by an Argon laser at $10-12 \mathrm{~W}$ of power. After placing the probe within the lumen of the arterial segment and heating up the tip for $2-3 \mathrm{sec}$, forward pressure was gently applied such that the tip could be observed just to begin to bend under fluoroscopy. The probe was kept acivated with gentle pressure applied for $5 \mathrm{sec}$ as it pressed against the obstruction. Intermittently, the catheter was pulled back and forth as it probed the obstruction with the laser activated. The laser was kept activated for a maximum of $10 \mathrm{sec}$ in any continuous attempt.

Mechanical probe In eight isolated arterial segments and in two in situ arteries, mechanical recanalization was attempted with a $6 \mathrm{Fr}$. multi-purpose catheter and an 0.035 inch guidewire or with a balloon dilata- 


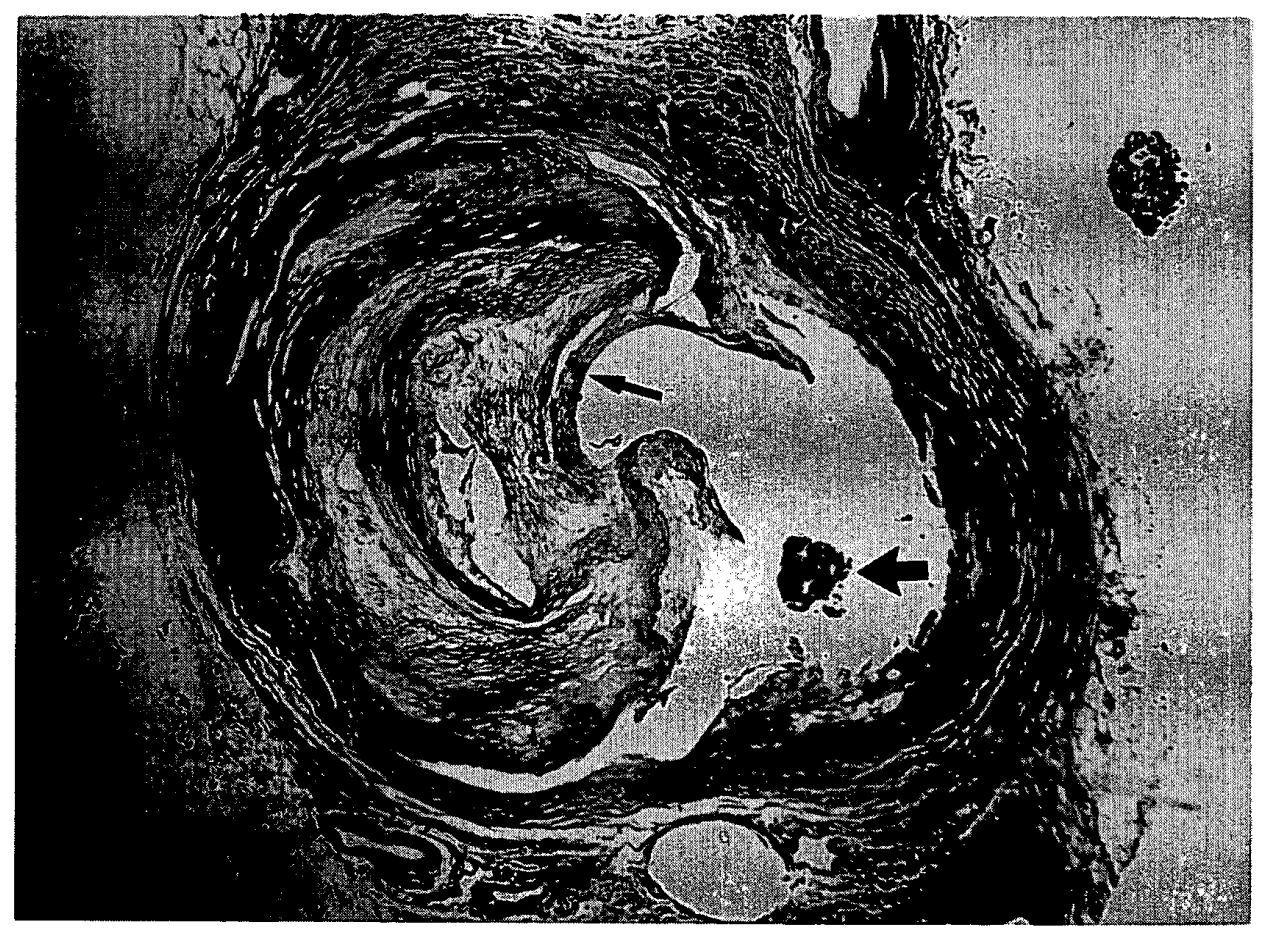

Fig.3. This cross-section demonstrates the consequence of the excimer laser radiation assisted by mechanical dilatation from the plastic catheter which supported the optic fiber. A large lumen was created as indicated by the pathway of the silk thread (thick arrow). No evidence of thermal injury is noted around the perimeter of the lumen created by the excimer radiation (thin arrow). However, the internal elastic membrane was torn and the atheroma was separated from the media as a result of mechanical dilatation with an $8 \mathrm{Fr}$ catheter.

tion catheter and an 0.018 inch guidewire.

The endpoint of each procedure was a successful exit through the distal end of the artery or lateral perforation of the arterial wall. After completion of the procedure, a 3.0 silk suture was pulled back through the newly created lumen and was left in place during histologic sectioning. The suture material was visible within the sections and permitted us to determine what lumen was created by each of the recanalization procedures in distinction to any residual natural lumen or artificial clefts produced by fixation. The artery segments were fixed in $10 \%$ formalin and stained with hematoxylin-eosin for subsequent light microscopic examination. Cross-sectional histologic examination was performed in each artery at $1 \mathrm{~cm}$ intervals from the proximal to the distal portion of the artery.

Statistical Methods The Chi-square statistic, adjusted for continuty, was used to compare the proportion of arteries with perforation among the three methods: excimer laser, thermal probe, and mechanical recana- lization. The Fisher's exact test was used for pairwise comparisons of perforation rates among the methods.

\section{RESULTS}

Excimer Laser Recanalization The excimer laser successfully recanalized 13 out of 21 $(62 \%)$ of the totally occluded arterial segments. A lateral perforation was observed in the remaining 8 arterial segments (38\%). In all of the perforated arteries, extensive calcified atheromas were noted.

Histologic examination demonstrated several characteristic findings produced by the excimer laser recanalization. Fig. 1 illustrates one of the typical patterns following excimer fiber recanalization. In this cross section, the excimer laser has created a new lumen into the central portion of the occlusive atheroma. The size of the newly created lumen is approximately the same as the size of the laser fiber ( 600 microns). There is no evidence of thermal injury at the perimeter of the lumen. 


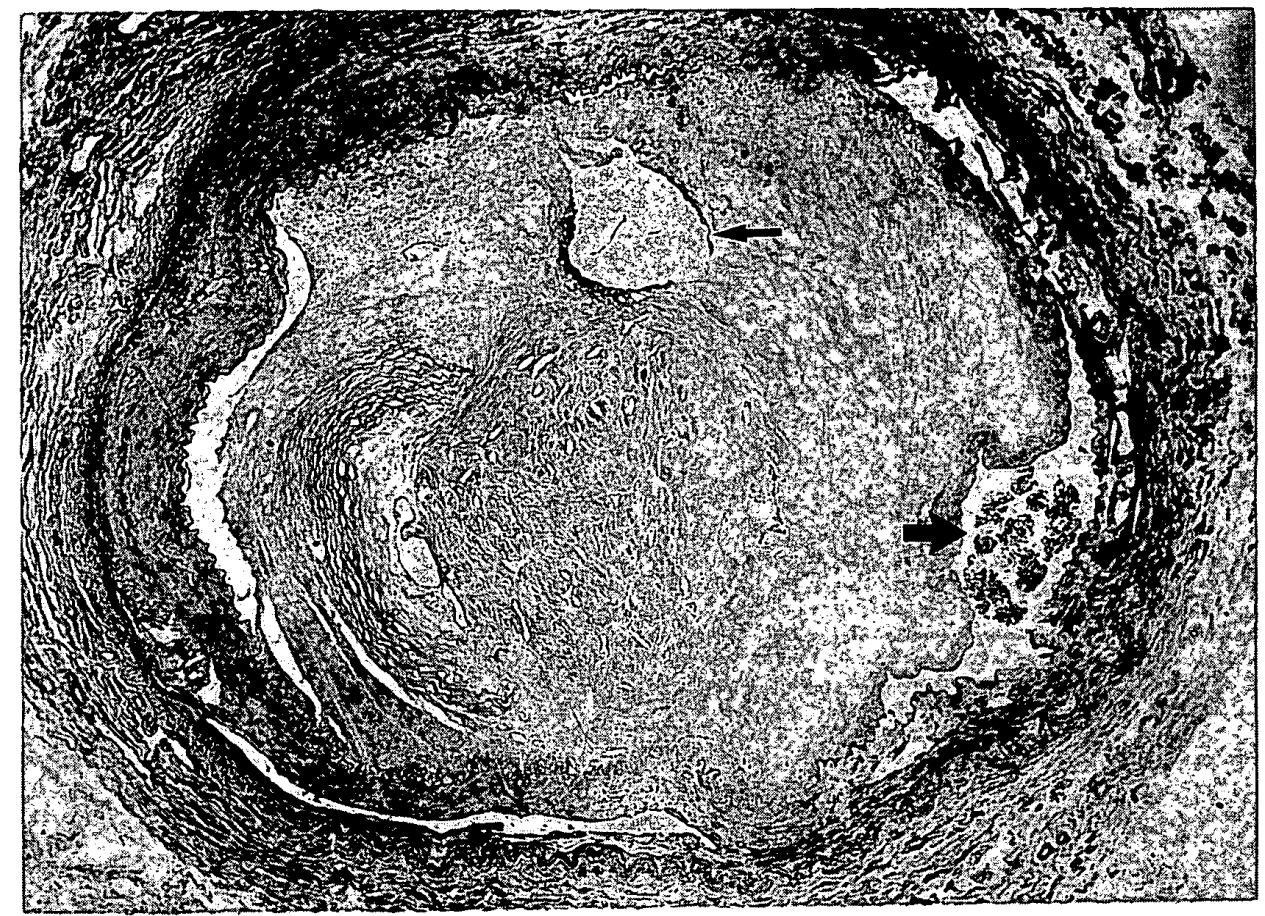

A

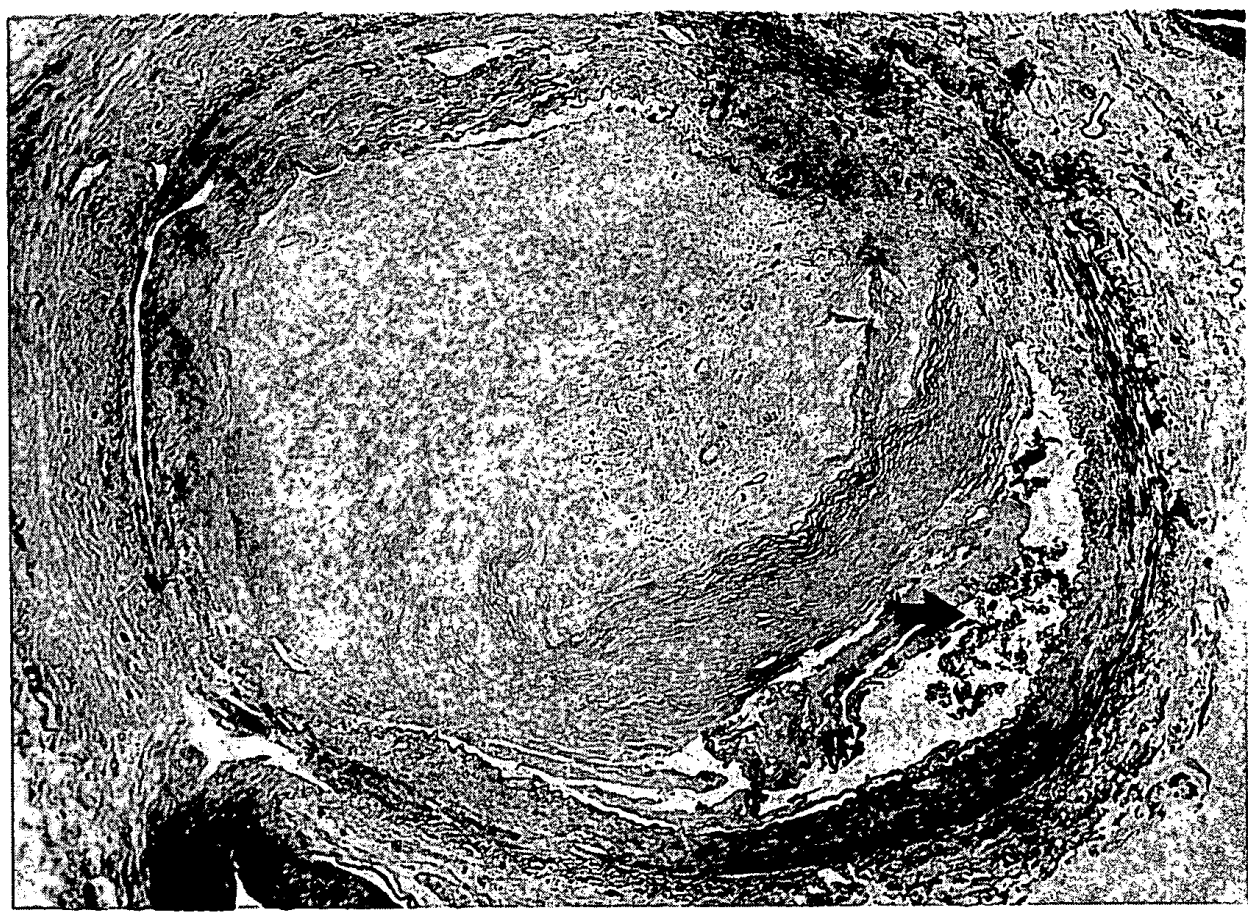

$\mathbf{B}$

Fig.4. Cross-sections of a totally occluded peripheral artery following excimer laser recanalization which demonstrate evidence of laser fiber deflection. In this artery, recanalization was performed twice. 4A) There are two paths from the 600 micron excimer laser fiber. One path is seen within the atheroma (thin arrow) and the other is between the intima and media documented by the silk thread (thick arrow). 4B) Cross-section of the same artery $3 \mathrm{~cm}$ distal to the section in 4A. Only one path is seen at this level because the two paths coalesced in this dissection plane (arrow) proximal to the internal elastic membrane. 


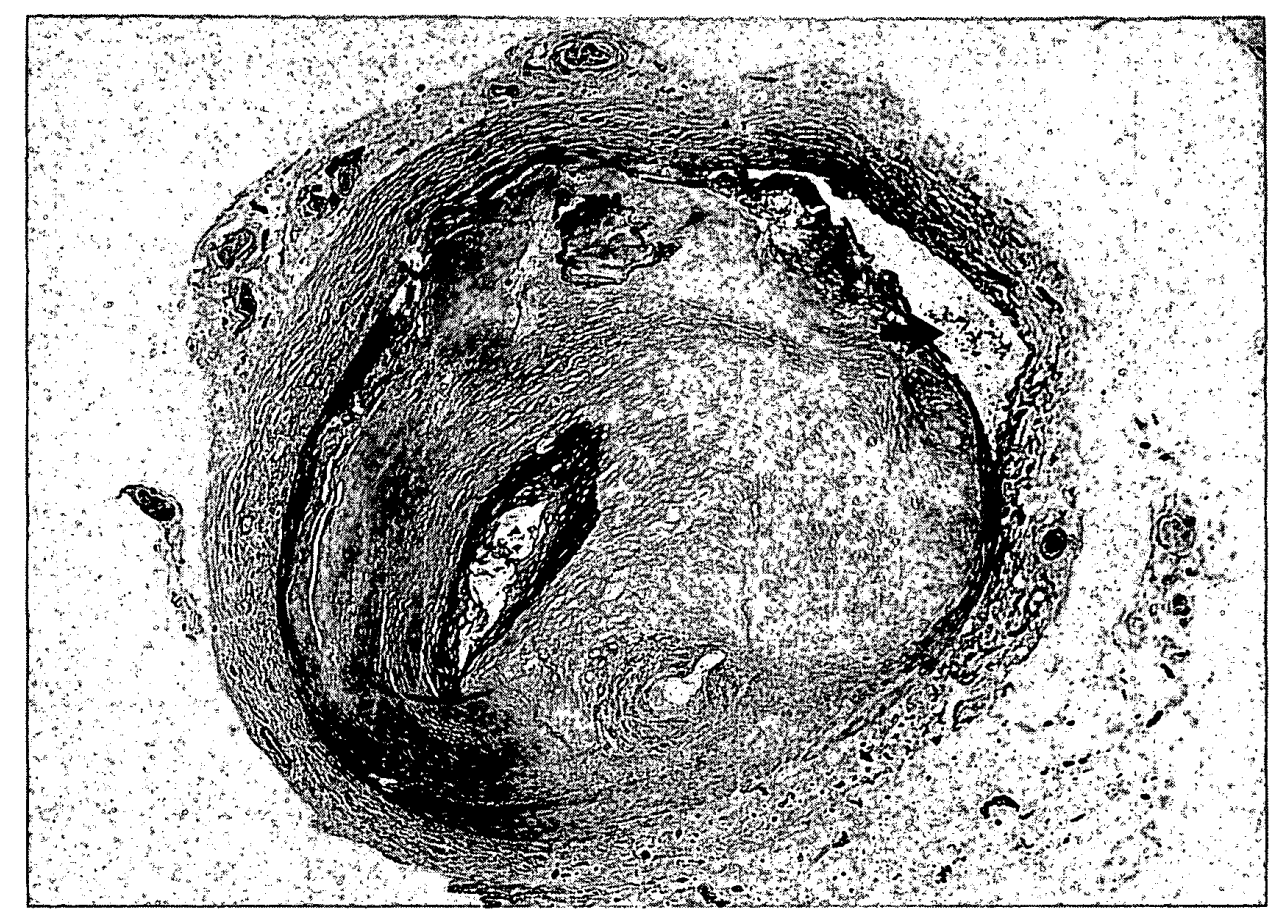

A

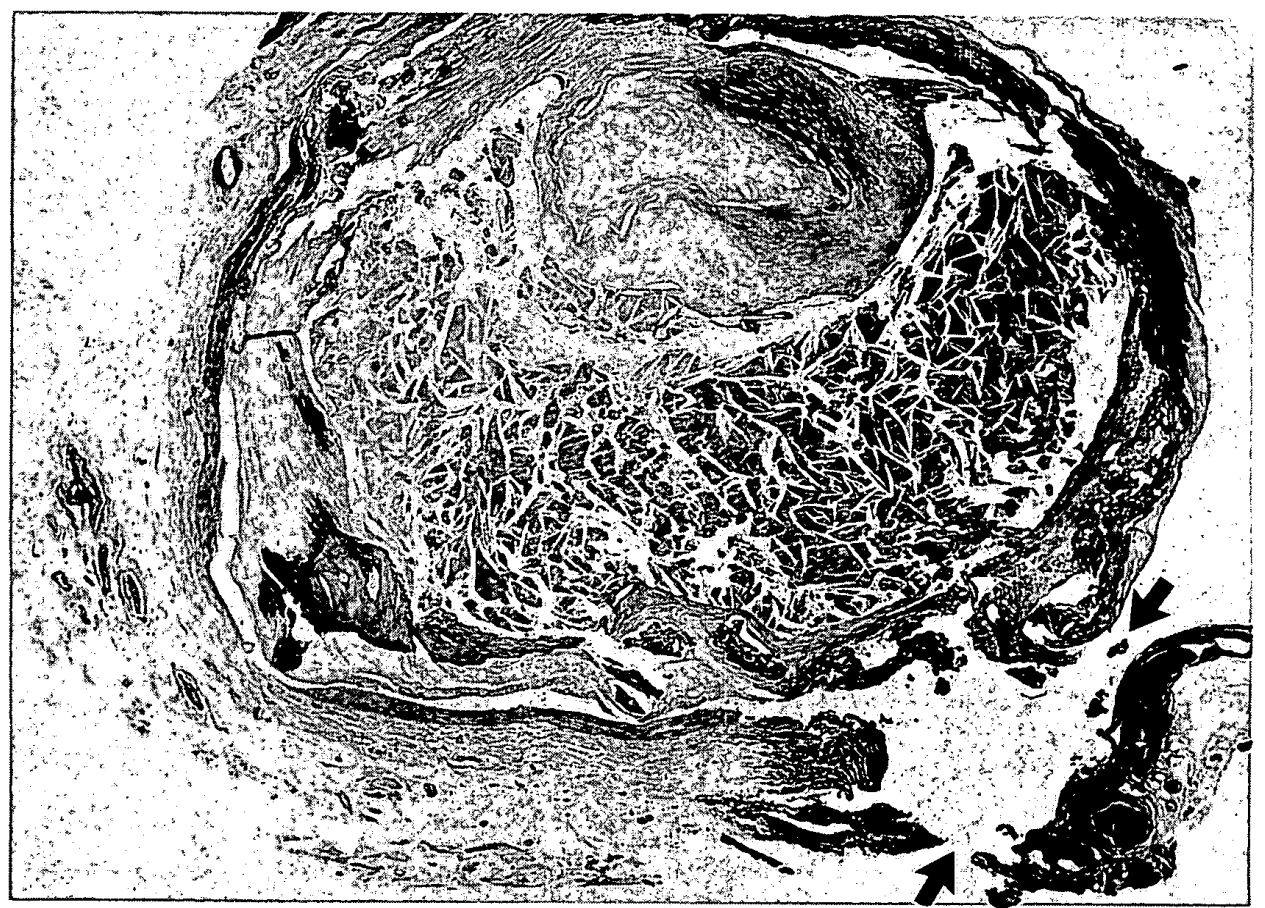

B

Fig.5. Cross-sections of a totally occluded human right coronary artery following thermal probe recanalization. 5A) The path of the probe documented by the silk thread (arrow) lies between the atheroma plaque and media. 5B) A perforation (arrows) is noted at the distal portion of the same artery. A circumferential thermal injury is evident.

Figure 2 demonstrates an ususal histologic finding caused by the excimer laser radiation which was not found with the thermal probe or mechanical devices. There are multiple lumens in this cross section which create a pattern similar to Swiss cheese. This pattern was created by moving the fiberoptic back and forth several times through the arterial 


\section{PATHWAY OF DEVICES}

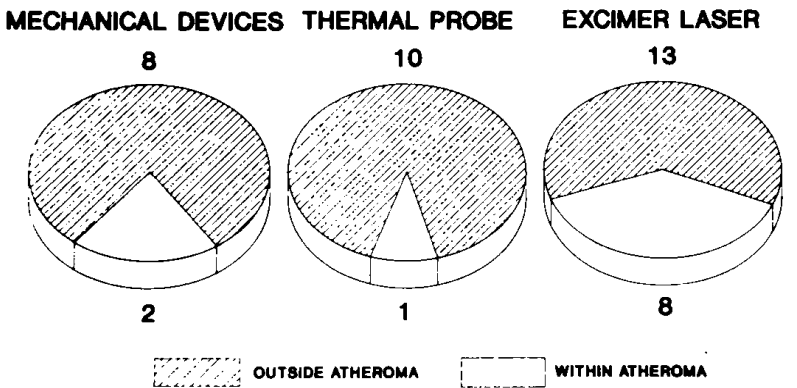

Fig.6. Pathway of the devices-Most of the devices were observed to pass outside of the central atheroma along the internal elastic membrane in all groups. The devices either remained in this dissection plane or eventually perforated the lateral wall.

segment. There is no thermal injury around the lumens and also no evidence of mechanical tears in this cross section. This multiple-lumen pattern was observed in two arterial segments.

Figure 3 documents the histologic consequence of an excimer laser recanalization assisted with mechanical dilatation. In this arterial segment, the excimer laser fiber was encased in an $8 \mathrm{Fr}$ catheter. There is no evidence of thermal injury around the lumen created by the excimer laser catheter. However, the atheroma plaque is torn with separation of the internal elastic membrane and the media. The splitting of the internal elastic membrane and the media occurred as a result of mechanical dilation by the catheter.

\section{PATHWAY OF THE DEVICES}

Excimer laser catheter The pathway that the various devices took was reconstructed by following the pathway of the silk thread in the histologic sections from the proximal to the distal end of the arterial segments. The excimer laser fiber passed through and remained within the atheroma for the length of the arterial segment in only 8 out of 21 $(38 \%)$ arterial segments. In the remaining 13 arterial segments $(62 \%)$ the laser fiber was deflected by the resistant atheromatous plaque and went along a dissection plane between the internal elastic membrane and the media. From this point, the laser fiber either remained within the dissection plane until it exited the distal end of the arterial segment

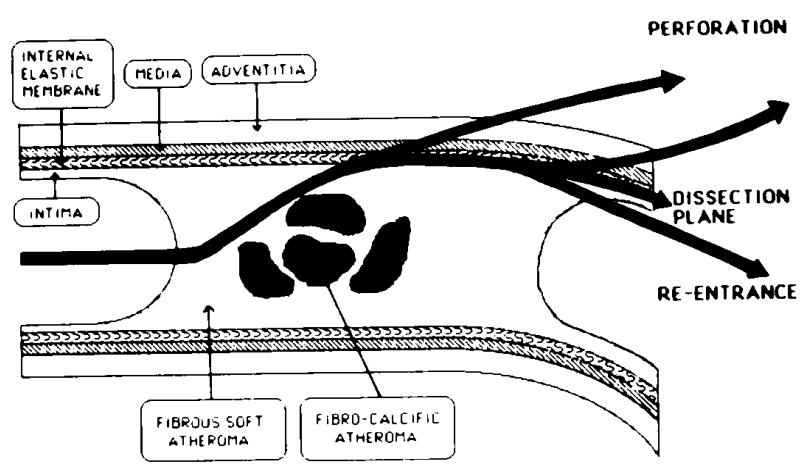

SCHEMAIIC DIAGRAM OF LASER CATHETER PATHWAYS

Fig.7. Schematic diagram of the laser fiber and catheter pathways. When there is a densely calcified or fibrotic atheroma, the laser catheters are deflected around the atheroma and advance into a less resistant dissection plane between the internal elastic membrane and the atheroma plaque. Eventually these devices may reenter the native lumen when the atheroma becomes thinner; they may remain within the dissection plane; or they may perforate externally.

or the fiber continued its tangental orientation and perforated the lateral wall of the artery. The typical pathway taken by the excimer fiber is represented in Fig. 4. The excimer laser was passed twice in this artery. These are two paths from the 600 micron excimer laser fiber in the proximal portion of the artery (Fig. 4A). One path is seen in the atheroma and the other path is between the intimal plaque and the internal elastic membrane just central to the media. In Fig. 4B, which was taken $3 \mathrm{~cm}$ distal to $4 \mathrm{~A}$, only one pathway is observed at the point where the more central fiber pathway through the atheroma has proceeded tangentally and has now entered the dissection plane between the internal elastic membrane and the atheroma. Once the fiberoptic catheter was in this peri-intimal dissection plane, the catheter was deflected by the atheroma and perforated externally in 8 of 13 arteries (62\%). If one includes the 8 arteries where the fiber remained within the atheroma, the total perforation rate was $38 \%$ ( 8 of $21 \mathrm{seg}$ ). In 5 of the 13 arteries $(38 \%)$, the excimer fiber continued to remain in the dissection plane and exited the distal end of the artery segment still within this plane and without perforating the media or adventitia. Moderate to severe arterial calcification was present in 15 of the 
$21 \mathrm{seg}$. Of these 15 calcified arteries, perforation occurred in $8(53 \%)$ cases. Thus all of the perforations occurred in arteries that were calcified and no perforation occurred in arteries that were not calcified.

Thermal laser probe As previously reported, the thermal probe deflected off the resistant atheroma and advaced in the plane between the intimal plaque and the internal elastic membrane in 10 out of 11 arteries $(91 \%) !^{10}$ The thermal probe advanced through the more central portion of the plaque and remained within the atheroma for the duration of the arterial segment in only one artery. An example of the pathway taken by the laser probe in a human coronary is demonstrated in Fig. 5. In each case where the thermal probe dissected between the atheroma and the media, the external wall was eventually perforated (perforation rate $=91 \%$ )

Mechanical devices In the majority of cases ( 8 out of $10,80 \%$ ) the guidewire and standard catheter did not remain within the atheroma plaque during recanalization. The pathway of the mechanical devices was observed to be within the dissection plane between the intima and the media, which was similar to the pathway taken by the laser probe or excimer catheter. Lateral perforation of the arterial wall was noted in two arteries, for a perforation rate of $20 \%$. A summary of the pathway taken by the mechanical devices, thermal probe, or excimer laser' catheter is provided in Fig. 6. For the purpose of these charts, the distinction was made whether the device remained within the atheroma plaque itself (whether or not if was centrally located) versus if the pathway was on the periphery of the atheroma in the dissection plane between the internal elastic membrane and the atheroma plaque. In the majority of cases for each of the techniques, the device was deflected by the reisistant atherosclerotic plaque and passed along the internal elastic membrane.

Comparison of perforation rates and pathway of recanalization The proportion of arteries in which perforation occurred differed significantly $\left(\mathrm{X}_{2}=12.1, \quad \mathrm{p}<0.01\right)$ according to the technique used for recanalization. The perforation rate by the excimer laser method $(8 / 21)$ was significantly lower $(\mathrm{p}<0.01)$ than for the thermal method
(10/11). The perforation rate for the mechanical method $(2 / 10)$ was also significantly lower $(p<0.02)$ than that of the thermal method. Rates of perforation between the excimer laser and mechanical methods, however, were not significantly different. There were also no significant differences among the three methods in the pathway taken by the intervention. The proportion of arteries in which there was deflection outside the atheroma but within the internal elastic membrane was not significantly different $\left(\mathrm{X}_{2}=3.4, \mathrm{p}=0.18\right)$ among those with excimer laser $(13 / 21)$, thermal probe $(10 / 11)$, or mechanical $(8 / 10)$ recanalization.

\section{DISCUSSION}

Determination of the various pathway that the guidewire, thermal probe, and excimer laser catheter take is important in understanding the mechanism of recanalization of occluded atherosclerotic arteries. It was observed that mechanical guidewires and the laser-heated thermal probe usually followed a similar pathway in that they were deflected from hard atheroma and advanced through a dissection plane between the internal elastic membrane and the intimal plaque $!^{0}$ This is the same dissection plane that is created during an endarterectomy. A schematic diagram of the pathways observed with a mechanical guidewire, thermal probe, and excimer laser catheter is demonstrated in Fig. 7. Once the device enters the dissection plane between the media and atheroma, it may either perforate laterally or may remain within the dissection plane along the internal elastic membrane, or the device may re-enter the distal lumen where the atheroma mass thins out proximal to the internal elastic membrane.

These findings suggest that the primary mechanism of thermal porbe recanalization may be a mechanical process. In this current study with the excimer laser fiber, it was found that the pathway of the excimer catheter was peripheral to the atheroma and proceeded along the dissection plane on approximately $2 / 3$ of the occluded arteries. This implies that recanalization with the excimer laser fiber also has a strong mechanical component which forces the laser fiber catheter away from hard plaque. Although the path- 
way of the excimer fiber was not significantly different from that of the thermal probe, the incidence of perforation was less than with the thermal probe. It is possible that the lower incidence of perforation in vitro with the excimer laser catheter may also be observed during future clinical trials. One reason for this difference in perforation rate may be understandable by following the pathway of the devices. Once the device enters the dissection plane between the internal elastic membrane and the atherosclerotic plaque, it might be expected that a $200{ }^{\circ} \mathrm{C}$ thermal probe would be more likely to burn laterally through the thin media and adventitia than would the non-thermal guidewires or excimer laser fibers. In this artificial in vitro model, once the thermal probe advanced in the dissection plane, a lateral perforation was eventually produced in all arteries $(10 / 10,100 \%)$. Thus, a higher incidence of perforation was noted with the thermal probe than with the excimer fiber $(10 / 11$, $91 \%$ vs $8 / 21,38 \% ; p<0.01)$. The difference in perforation rate also could be explained if there were inherent differences in the resistance of the plaque in the arteries used for the excimer laser studies compared to the other devices. The artery samples were not randomly assigned to the three techniques. However, the intent of the study was not to quantitate the number of times that the devices perforated as much as to identify the pathway that the catheter or laser fiber takes during a successful recanalization and to help understand the process of perforation when it occurs. The incidence of perforation in this study with mechanical guidewires is higher than that experienced clinically. This result may be due to the relatively long occlusions and the lack of supporting tissues around the artery in this in vitro study. Because the occlusion length with the excimer fiber was significantly shorter than those with the mechanical guidewire and thermal probe, it is also possible that the perforation rate is influenced by the occlusion length. However, this was not a major factor because the occlusion length did not differ significantly among those with or without perforation $(5.1$ vs $4.4 \mathrm{~cm})$. A larger randomized study comparing perforation rates and recanalization pathways among different methods of intervention (e.g., excimer laser, thermal probe, and mechanical techniques) is recommended to confirm these findings.

The histologic study of human occluded arteries recanalized with excimer laser energy demonstrated several characteristic findings. When the optic fiber was used alone, a small lumen was created by the excimer laser radiation. The rim of the lumen was extremely smooth without evidence of thermal damage. This could have potential advantage in the clinical setting if this observation corresponded to a less thrombogenic surface. The small lumen created by the $400-800$ micron fiber would be inadequate for sufficient blood supply to the distal artery. Our observations in vitro suggest that the larger lumens that were seen on histology were due to the mechanical stretching and tearing of the atherosclerotic plaque by the guiding catheter that was glued to the optic fiber. Another unusual finding on the histologic sections was the multiple holes or Swiss cheese-like appearance which was obtained by passing the excimer laser fiber slowly back and forth through the arterial segment. The precise mechanism of this phenomenon is uncertain, but a possible explanation is that small changes in the direction of the laser beam during the procedure, especially when the fiber encountered a resistant atheroma, is likely to cause variations in the direction of the laser beam. This multiple hole pattern did not seem to influence the success of the recanalization attempt.

\section{CONCLUSION}

The major observations from this in vitro study are (1) that an excimer laser fiber catheter can recanalize occluded human atherosclerotic arteries without producing thermal injury; (2) the size of the created lumen was relatively small, but enough to pass a guidewire for adjunctive mechanical dilatation; (3) the incidence of perforation was less with an excimer laser fiber catheter than with a thermal probe. However, When the obstruction was composed of a hard, fibrocalcific plaque, the excimer laser catheter was frequently deflected around the atherosclerotic plaque and passed along the plane between the intimal plaque and the media. This pathway occurs similarly with mechanical guidewires or the thermal probe. 
These results suggest that the mechanism of excimer laser recanalization of human arteries depends to a large extent on mechanical proccesses. These observations imply that to prevent perforation during recanalization of occluded arteries with bare laser radiation, it will be necessary to develop a better guidance system.

\section{REFERENCES}

1. GINSBURG R, WEXLER L, MITCHELL RS, PROFITT D: Percutaneous transluminal laser angioplasty for treatment of peripheral vascular disease: Clinical experience with 16 patients. Radiology 156: 619, 1985

2. CUMBERLAND DC, SANBORN TA, TAYLER DI, MOORE DJ, WELSH CL, GREENFIELD AJ, GUBEN JK, RYAN TJ: Percutaneous laser thermal angioplasty: Clinical results with a bare laser probe in total peripheral occlusions. Lancet 1: 1457,1986

3. SANBORN TA, FAXON DP, KELLET MA, RYAN TJ: Percutaneous coronary laser angioplasty. J Am Coll Cardiol 8: 1437, 1986

4. KATZEN BT, SCHWARTEN DM, KAPLAN JO, CUTCLIFF W: Initial experience with an excimer laser in peripheral lesions. Circulation 78(II): 417, 1988

5. LITVACK $F$, GRUNDFEST $W$, ADLER L, HICKEY A, SEGALOWITZ J, HESTRIN L, GOLDBERG T, LAUDENSLAGER J, FORRESTER J: Percutaneous excimer laser angioplasty in humans. Circulation 78(II): 295, 1988

6. ABELA GS, NORMANN S, COHEN D, FELDMAN RL, GEISER EA, CONTI CR: Effects of carbon dioxide Nd-YAG and argon laser radiation on coronary atheromatous plaque. Am J Cardiol 50: 1199,1982

7. SANBORN TA, FAXON DP, HAUDENSHILD CC, RYAN TJ: Experimental angioplasty: Circumferential distribution of laser thermal energy with a laser probe. J Am Coll Cardiol 5: 934, 1985

8. GARRISON BJ, SRINIVASAN R: Microscopic model for the ablative photodecomposition of polymers by far ultraviolet radiation $(196 \mathrm{~nm})$. Appl Phys Lett 44(9): 849-51, 1984

9. BRANON JH, LANKARD JR, BAISE AI, BURNS F, KAUFMAN J: Excimer laser etching of polyimide. J Appl Phys 58(5): 2036, 1985

10. TOBIS JM, SMOLIN M, MALLERY JA, MACLEAY L, JOHNSTON WD, CONNOLLY JE, LEWIS G, ZUCH R, HENRY WL, BERNS M: Laser assisted thermal angioplasty in human peripheral artery occlusions: Mechanism of recanalization. J Am Coll Cardiol 13: 1547, 1989

11. GRUNDFEST WS, LITVACK F, FORRESTER JS, GORDBERG T, SWAN HJC, MORGENSTERN L, FISHBEIN M, Mc DERMID IS, RIDER DM, PACALA TJ, LAUDENSLAGER JB: Laser ablation of human atherosclerotic plaque without adjacent tissue injury. J Am Coll Cardiol 5: 929,1985

12. ISNER JM, DONALDSON RF, DECKELBAUM LI, CLARKE RH, LALIBERTE SM, UCCI AA, SALEM DN, KONSTAM MA: The excimer laser: Gross, light microscopic and ultrastructural analysis of potential advantages for use in laser therapy of cardiovascular disease. J Am Coll Cardiol 6: 1102, 1985

13. DECKLBAUM LI, ISNER JM, DONALDSON RF, CLARKE RH, LALIBERTE S, AHARON AS, BERNSTEIN JS: Reduction of laser induced pathologic tissue injury using pulsed energy delivery. Am J Cardiol 56: 663, 1985 